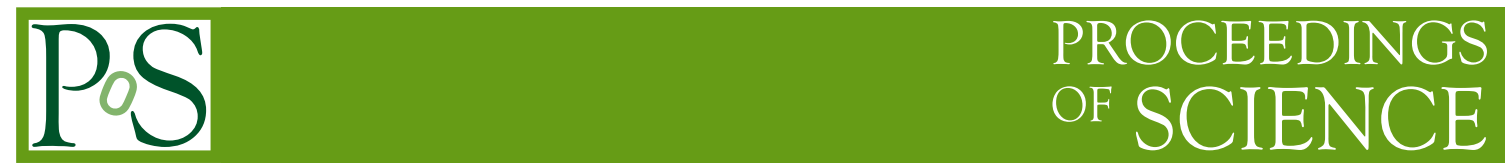

\title{
Perturbative probes of QCD matter at the LHC
}

\author{
David d'Enterria* \\ CERN, PH Department, 1211 Geneva, Switzerland \\ E-mail: david.d'enterria@cern.ch
}

The main results on electroweak probes, jets, high- $p_{T}$ hadrons, heavy-flavour and quarkonia production from the first two years of heavy-ion operation at the Large Hadron Collider (LHC) are briefly reviewed. Data measured at center-of-mass energies $\sqrt{s_{N N}}=2.76 \mathrm{TeV}$ in lead-lead $(\mathrm{Pb}-\mathrm{Pb})$ collisions are compared to proton-proton (p-p) measurements in order to extract information on the properties of hot and dense strongly-interacting matter.

6th International Conference on Quarks and Nuclear Physics

April 16-20, 2012

Ecole Polytechnique, Palaiseau, Paris

${ }^{*}$ Speaker. 


\section{Introduction}

Nucleus-nucleus collisions (A-A) at ultrarelativistic energies provide the experimental means to study the (thermo)dynamics of quarks and gluons under extreme conditions of temperature and density. Head-on collisions of $\mathrm{Pb}$ ions at LHC energies produce very hot and dense matter by concentrating a substantial amount of energy $\mathscr{O}(2 \mathrm{TeV})$ in an extended volume $\mathscr{O}\left(150 \mathrm{fm}^{3}\right)$ at thermalisation times of $\tau_{0}=1 \mathrm{fm} / \mathrm{c}$ [1]. Such energy densities are more than one order of magnitude above the critical value, $\varepsilon_{\text {crit }} \approx 1 \mathrm{GeV} / \mathrm{fm}^{3}$, predicted by lattice quantum chromodynamics (QCD) calculations [2] for the formation of a deconfined system of bare-mass quarks and gluons (Quark Gluon Plasma, QGP) [3]. Among all experimental observables, particles with large transverse
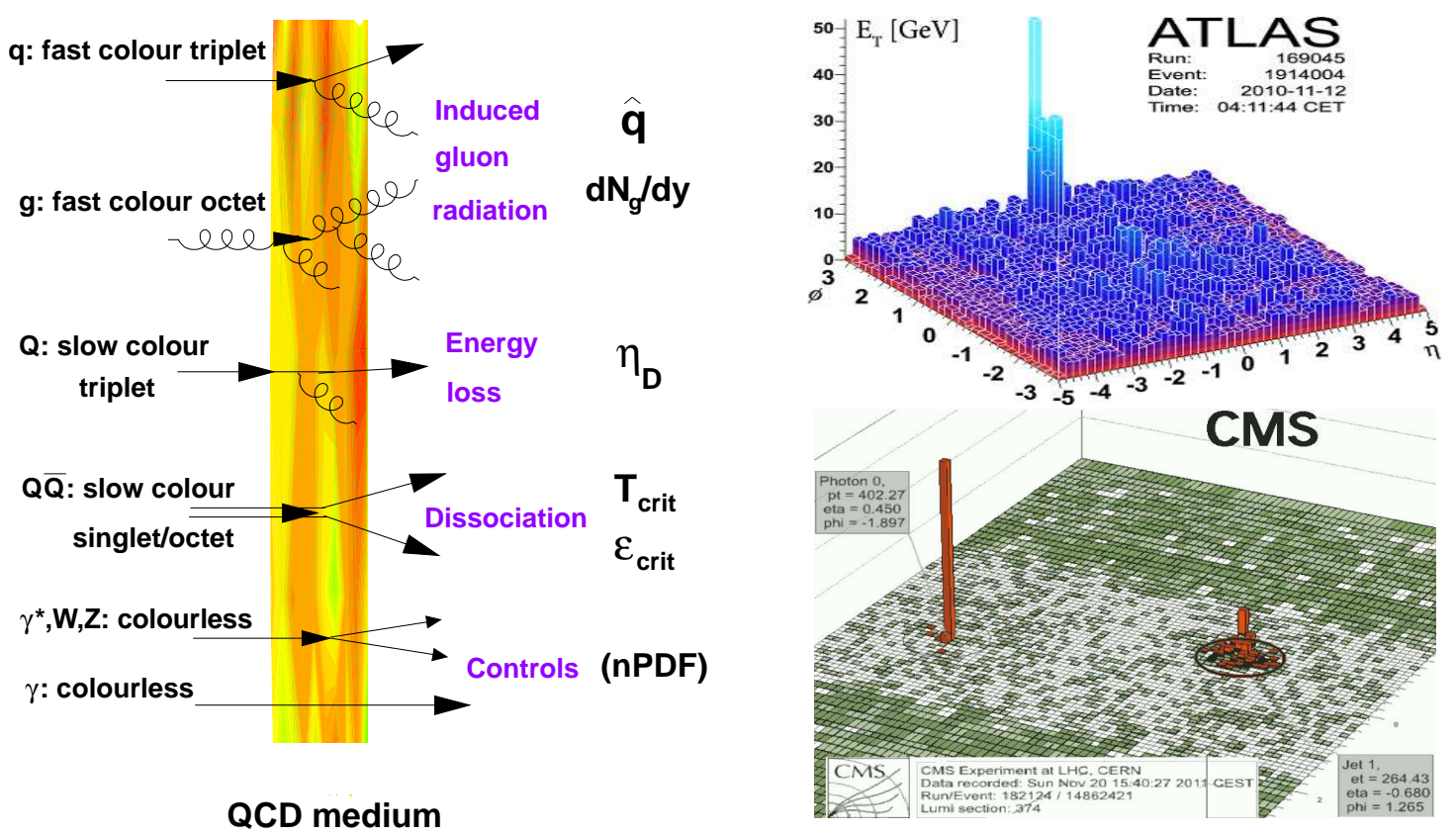

Figure 1: Left: Examples of the sensitivity of various perturbative probes to quark-gluon matter properties in A-A collisions [4]. Right: "Jet quenching" event displays in central Pb-Pb collisions at the LHC: monojetlike event [5] (top) and $\gamma$-jet event with a recoiling jet with reduced energy [6] (bottom).

momentum $p_{T}$ and/or high mass $m$ ("hard probes") are useful tomographic tools of the produced medium (Fig. 1 left) as: (i) they originate from partonic scatterings with large momentum transfer $Q^{2}$ and couple directly to the fundamental quark and gluon degrees of freedom; (ii) their production time-scale is very short, $\tau \approx 1 /\left(p_{T}, m\right) \lesssim 0.1 \mathrm{fm} / \mathrm{c}$, allowing them to propagate through (and be potentially affected by) the medium, and (iii) their cross sections can be theoretically computed in perturbative QCD calculations as a convolution of parton distribution (PDFs, $f_{a / A}$ ) and fragmentation (FFs, $D_{c \rightarrow h}$ ) functions times the subprocess parton-parton scattering cross section: $d \sigma_{A B \rightarrow h}^{\text {hard }}=$ $f_{a / A}\left(x, Q^{2}\right) \otimes f_{b / B}\left(x, Q^{2}\right) \otimes d \sigma_{a b \rightarrow c}^{\text {hard }} \otimes D_{c \rightarrow h}\left(z, Q^{2}\right)$. In A-A collisions in the absence of final-state effects, the parton flux in a nucleus $A$ is the same as that of a superposition of $A$ independent nucleons, $f_{a / A} \approx A \cdot f_{a / N}$, and thus $d \sigma_{A A \rightarrow h}^{\text {hard }} \approx A^{2} \cdot f_{a / N}\left(x, Q^{2}\right) \otimes f_{a / N}\left(x, Q^{2}\right) \otimes d \sigma_{a b \rightarrow c}^{\text {hard }} \otimes D_{c \rightarrow h}\left(z, Q^{2}\right)$. The standard method to quantify the effects of the medium on a given hard probe is via the ratio of A-A yields over p-p cross sections (scaled by the nuclear overlap function $T_{A A}(b)$ at impact parameter $b$ ): $R_{A A}\left(p_{T}, y ; b\right)=\frac{d^{2} N_{A A} / d y d p_{T}}{\left\langle T_{A A}(b)\right\rangle \times d^{2} \sigma_{p p} / d y d p_{T}}$, which measures the deviation of A-A at $b$ from an incoherent 
superposition of nucleon-nucleon collisions. The study of the suppression (or enhancement) factors as a function of various variables provides information on the medium transport coefficients, its temperature, energy density, etc. (Fig. 1, left).

\section{Unsuppressed electro-weak particle production}

Weakly-interacting particles such as isolated photons, W, Z, and Drell-Yan pairs - whose rates are unaffected by final-state interactions in the produced medium - are valuable benchmark processes in A-A collisions at collider energies. On the one hand, they allow one to experimentally confirm the validity of the perturbative $\left(A^{2}\right)$ scaling of the p-p cross sections and, on the other, they provide constraints on the nuclear PDFs which, in particular for the $\mathrm{Pb}$ case, are barely known from deep-inelastic e-A data [7]. At the LHC, prompt- $\gamma$ have been measured above $20 \mathrm{GeV} / \mathrm{c}$ applying isolation cuts on background-subtracted $\mathrm{Pb}-\mathrm{Pb}$ collisions [8], $\mathrm{Z}$ bosons have been measured in the dimuon $[9,10]$ and dielectron $[11]$ decay modes, and $\mathrm{W}$ bosons in events with a high- $p_{T}$ muon and large missing transverse momentum from the undetected $v$ [12] (Fig. 2). In all three cases,
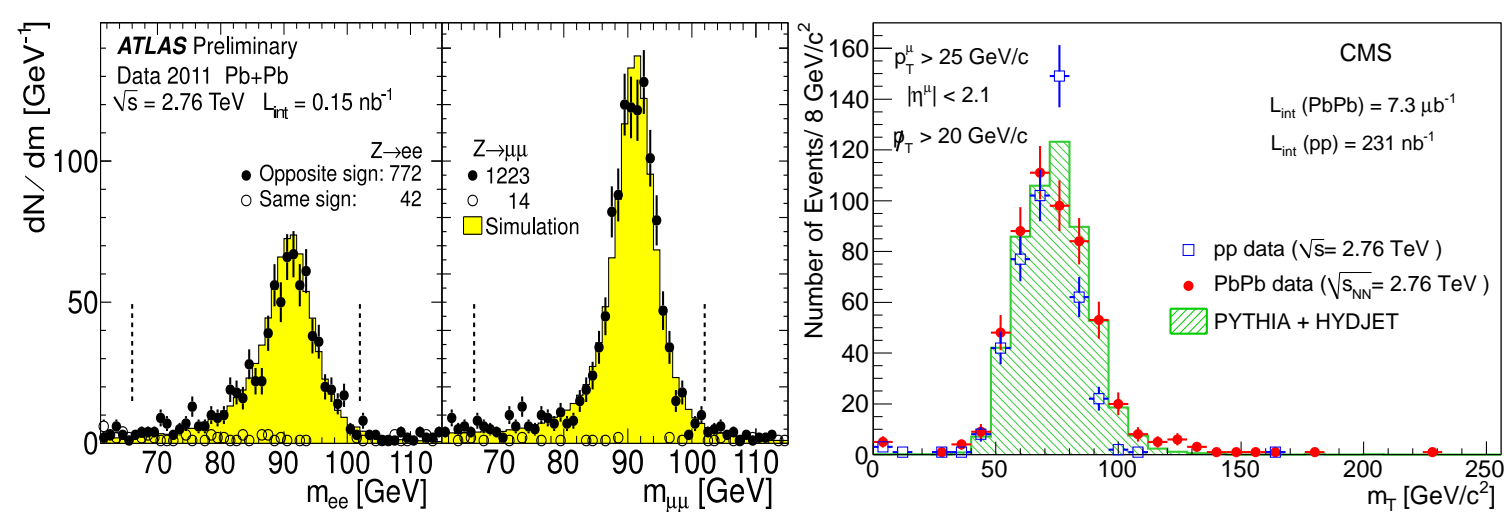

Figure 2: Measured mass distributions for $\mathrm{Z}$ bosons $\left(\mu^{+} \mu^{-}\right.$and $e^{+} e^{-}$invariant masses, left) [11] and $\mathrm{W}$ (transverse mass, right) [12] in $\mathrm{Pb}-\mathrm{Pb}$ (and $\mathrm{p}-\mathrm{p}$ ) collisions at $2.76 \mathrm{TeV}$.

the measured yields for all $\mathrm{Pb}-\mathrm{Pb}$ centralities are consistent with the corresponding $\mathrm{p}$-p cross sections scaled by the nuclear overlap function $\left(R_{A A} \approx 1\right.$, Fig. 3 left). Comparisons to NLO pQCD calculations indicate small nuclear PDF modifications as expected in the ranges of parton fractional momentum $x$ and energy scale $Q^{2}$ probed [7]. Interestingly, inclusive W production shows $R_{A A}(\mathrm{~W})=1.04 \pm 0.07 \pm 0.12$ while individual $\mathrm{W}^{ \pm}$yields show differences due to isospin effects (unequal $u$ and $d$-quark content in $\mathrm{Pb}$ and p): $R_{A A}\left(\bar{u} d \rightarrow \mathrm{W}^{-}\right)=1.46$ and $R_{A A}\left(u \bar{d} \rightarrow \mathrm{W}^{+}\right)=0.8$ [12]. Future analyses of larger $\gamma, \gamma^{\star}, \mathrm{W}, \mathrm{Z}$ data samples with reduced uncertainties, will provide enhanced constraints on the parton densities in nuclei.

\section{Suppression of high- $p_{T}$ hadrons}

One of the first proposed smoking guns of QGP formation was "jet quenching" [13] i.e. the attenuation or disappearance of the spray of hadrons resulting from the fragmentation of a parton having lost energy in the dense plasma produced in the reaction. Among the most exciting results from RHIC is the observation of large high- $p_{T}$ hadron suppression $\left(R_{A A} \approx 0.2\right)$ in central $\mathrm{Au}-\mathrm{Au}$ 
compared to p-p or d-Au collisions, in agreement with jet quenching expectations [14, 15]. At the LHC, charged hadrons appear to be further suppressed, by up to factor of 6 at $p_{T}=7 \mathrm{GeV} / \mathrm{c}[16,17]$ but the amount of suppression is slowly reduced with increasing $p_{T}$ and plateaus at $R_{A A} \approx 0.5$ at $p_{T} \approx 40-100 \mathrm{GeV} / \mathrm{c}[18]$ (Fig. 3, left). The observed $p_{T}$ dependence provides strong discrimina-
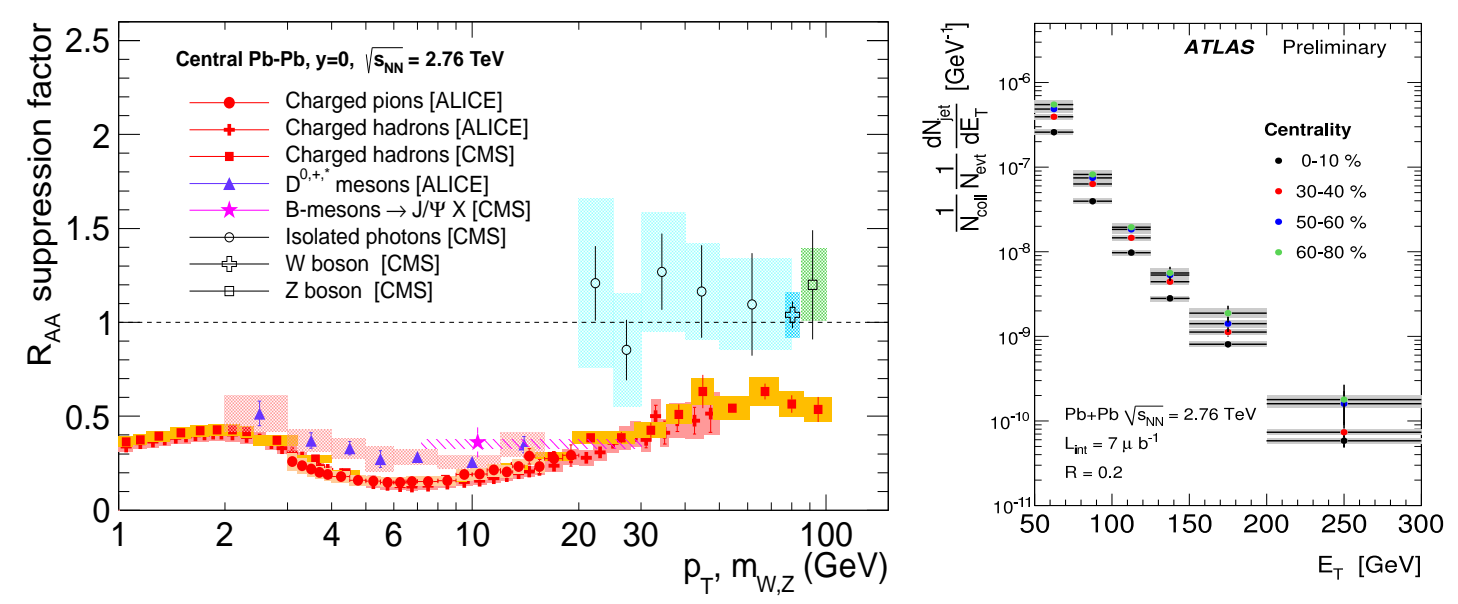

Figure 3: Left: Compilation of measured $R_{A A}\left(p_{T}\right)$ in central $\mathrm{Pb}-\mathrm{Pb}$ collisions at the LHC for inclusive charged hadrons [16, 18], $\pi^{ \pm}$[17], D [19] and B [20] mesons, isolated- $\gamma$ [8], and W [12] and Z [10] bosons. Right: Jet spectra (anti- $k_{T}$ algorithm, $R=0.2$ ) measured in various $\mathrm{Pb}-\mathrm{Pb}$ centralities scaled by their corresponding $T_{A A}$ [21]. The bands around all data points indicate the associated systematic uncertainties.

tion power for parton radiative energy loss models. Once the underlying energy loss mechanism responsible for the observed high- $p_{T}$ hadron deficit is confirmed [22] the properties of the medium (e.g. its $\hat{q}$ transport coefficient) can be properly derived taking into account the expansion of the produced matter in 3D-hydrodynamics calculations [23].

\section{Jet quenching in dijet and $\gamma$-jet events}

High- $p_{T}$ jets have been fully reconstructed in heavy-ion collisions for the first time at the LHC using modern algorithms for jet finding and background subtraction [24]. The strongest evidence so far for jet quenching is the observation of a large dijet momentum imbalance for increasingly central $\mathrm{Pb}-\mathrm{Pb}$ collisions $[5,25]$ up to very high momenta ( $p_{T} \approx 350 \mathrm{GeV} / \mathrm{c}$ ) [26]. In some cases, the quenched jet is not even visible above the underlying event background (Fig. 1, right). As observed for the inclusive (leading) hadrons above $40 \mathrm{GeV} / \mathrm{c}$, the jet spectra in central $\mathrm{Pb}-\mathrm{Pb}$ are suppressed by a factor of two compared to peripheral $\mathrm{Pb}-\mathrm{Pb}$ collisions (Fig. 3, right) [21]. The lost energy of the jet appears to be emitted in the form of soft particles $\left(p_{T}<4 \mathrm{GeV} / \mathrm{c}\right)$ outside of jet cone $(R>0.8)$ [25]. Interestingly, even if the quenched jet loses a large fraction of its energy, the dijet event keeps its back-to-back topology without significant azimuthal decorrelation [5, 25]. Fragmentation functions have been constructed using tracks with $p_{T}>2(4) \mathrm{GeV} / \mathrm{c}$ within $R<0.2(0.3)$ of the jet-axis combined with the reconstructed (but quenched) jet energy [21, 27]. The resulting FFs, shown in Fig. 4 as a function of the longitudinal fraction of the jet momentum carried by the charged particles $z=p_{\|}^{\text {track }} / p^{\text {jet }}$ (left) and of $\xi=\ln (1 / z)$ (center), are similar in $\mathrm{Pb}-\mathrm{Pb}$ (central and 
peripheral) and p-p collisions indicating that after energy loss the quenched jet fragments in the vacuum with the same pattern as "normal" jets. Jet quenching has also been studied in "cleaner"
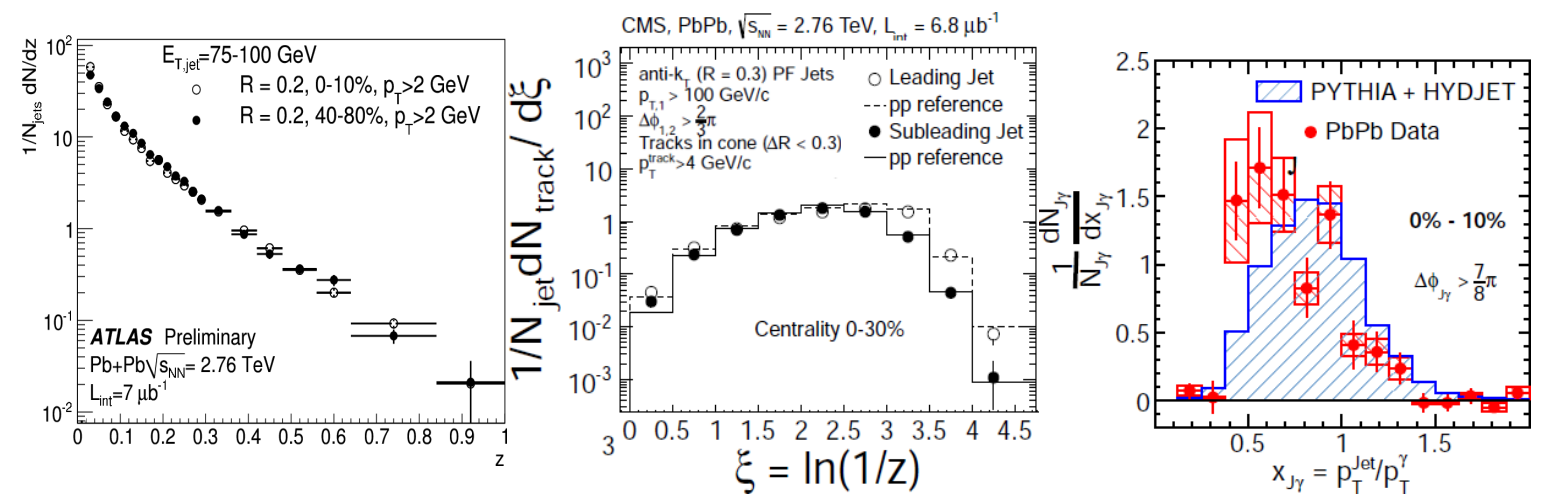

Figure 4: Jet fragmentation functions reconstructed in $\mathrm{Pb}-\mathrm{Pb}$ at $2.76 \mathrm{TeV}$ as a function of the scaling variables $z$ (left panel, for central and peripheral collisions) [21] and $\xi$ (mid panel, for central collisions) [27]. Right: Ratio of jet to photon momenta in central $\mathrm{Pb}-\mathrm{Pb}$ collisions compared to PYTHIA p-p predictions [6].

photon-jet events with $p_{T}^{\gamma}>60 \mathrm{GeV} / \mathrm{c}$ and $p_{T}^{\text {jet }}>30 \mathrm{GeV} / \mathrm{c}$ [6]. The balance ratio $x_{\mathrm{J} \gamma}=p_{T}^{\text {jet }} / p_{T}^{\gamma}$ is seen to decrease significantly with increasing centrality (see Fig. 4 right, for central $\mathrm{Pb}-\mathrm{Pb}$ ) as does the fraction of $\gamma$-jet associations found in the data, compared to p-p and PYTHIA [28] results. Yet, the $\gamma$-jet azimuthal distribution measured in $\mathrm{Pb}-\mathrm{Pb}$ events with large $p_{T}$ imbalance is consistent with that measured in p-p collisions and simulated with PYTHIA. The fact that the quenched jet is back-to-back to the photon confirms that the energy is not lost in single hard gluon radiation.

\section{Heavy-flavour suppression}

In QCD, gluon-strahlung from heavy quarks is expected to be less important than for light quarks due to the "dead-cone" effect [29]. Thus, a robust prediction of jet quenching models is the hierarchy $\Delta E_{Q}<\Delta E_{q}<\Delta E_{g}$ accounting for mass and colour-charge dependencies of radiative energy loss. Surprisingly, RHIC measurements of high- $p_{T}$ electrons from semileptonic $D$ and $B$ decays seem to indicate a charm+bottom suppression comparable to that of light quarks: $R_{A A}(c, b) \approx R_{A A}(q, g) \approx 0.2[30,31]$. At the LHC, D-mesons have been for the first time directly measured in $\mathrm{Pb}-\mathrm{Pb}$ events with displaced-vertices in three hadronic decay channels $\left(D^{0} \rightarrow K \pi\right.$, $D^{ \pm} \rightarrow K \pi \pi, D^{\star} \rightarrow D^{0} \pi$ for $p_{T}>2,4$ and $6 \mathrm{GeV} / \mathrm{c}$ respectively) [19], and B-meson production has been observed via secondary $J / \psi$ for which a clean separation of the displaced vertex is also possible for $p_{T}>6.5 \mathrm{GeV} / \mathrm{c}$ [20]. Within uncertainties, the measured suppression factors, $R_{A A}(\mathrm{D}) \approx 0.3$ and $R_{A A}(\mathrm{~B}) \approx 0.4$ are larger than $R_{A A}\left(\mathrm{~h}^{ \pm}, \pi^{ \pm}\right) \approx 0.15-0.2$ (Fig. 3, left) and support the expected radiative energy loss hierarchy. Coming measurements with larger data samples and reduced uncertainties will allow one to better understand the behaviour of heavy-quarks in the QGP, including the relative contribution of radiative/elastic losses, and determine e.g. their drag coefficients $\eta_{D}$ in the medium [32].

\section{Quarkonia dissociation}

The study of heavy-quark bound states in high-energy A-A collisions has a long story as a 
sensitive probe of the thermodynamical properties of deconfined QCD matter [33]. Analysis of quarkonia correlators and potentials in finite- $T$ lattice QCD [34] indicate that the different $c-\bar{c}$ and $b-\bar{b}$ states dissociate at temperatures for which the colour (Debye) screening radius of the medium falls below their corresponding $Q \bar{Q}$ binding radius. The surprisingly similar amount of $J / \psi$ suppression observed at SPS [35] and RHIC [36, 37] energies (with expected larger QGP temperature in the latter) has been interpreted as due to a partial compensation at RHIC of the reduced $J / \psi$ yields by $c \bar{c}$ recombination in the medium $[38,39]$. In this scenario, the large charm pair production expected at the LHC would further enhance charmonium regeneration [40]. LHC data on $J / \psi$ production $[9,20,41]$ show a different suppression pattern than observed at RHIC (Fig. 5, left). At low $p_{T}, J / \psi$ are less suppressed $\left(R_{A A} \approx 0.5\right)$ than measured at lower c.m. energies $\left(R_{A A} \approx 0.2-\right.$ $0.3)$, whereas at high- $p_{T}$ they are more suppressed $\left(R_{A A} \approx 0.2\right)$ than at RHIC $\left(R_{A A} \approx 0.6\right)$. Approaches based on deconfinement followed by charm recombination can reproduce the observed trends in the data although the model parameters $\left(\sigma_{c \bar{c}}\right.$, density, ...) need to be validated with other LHC observations. The abundant production of the $\Upsilon(1 S, 2 S, 3 S)$ states at the LHC opens up a
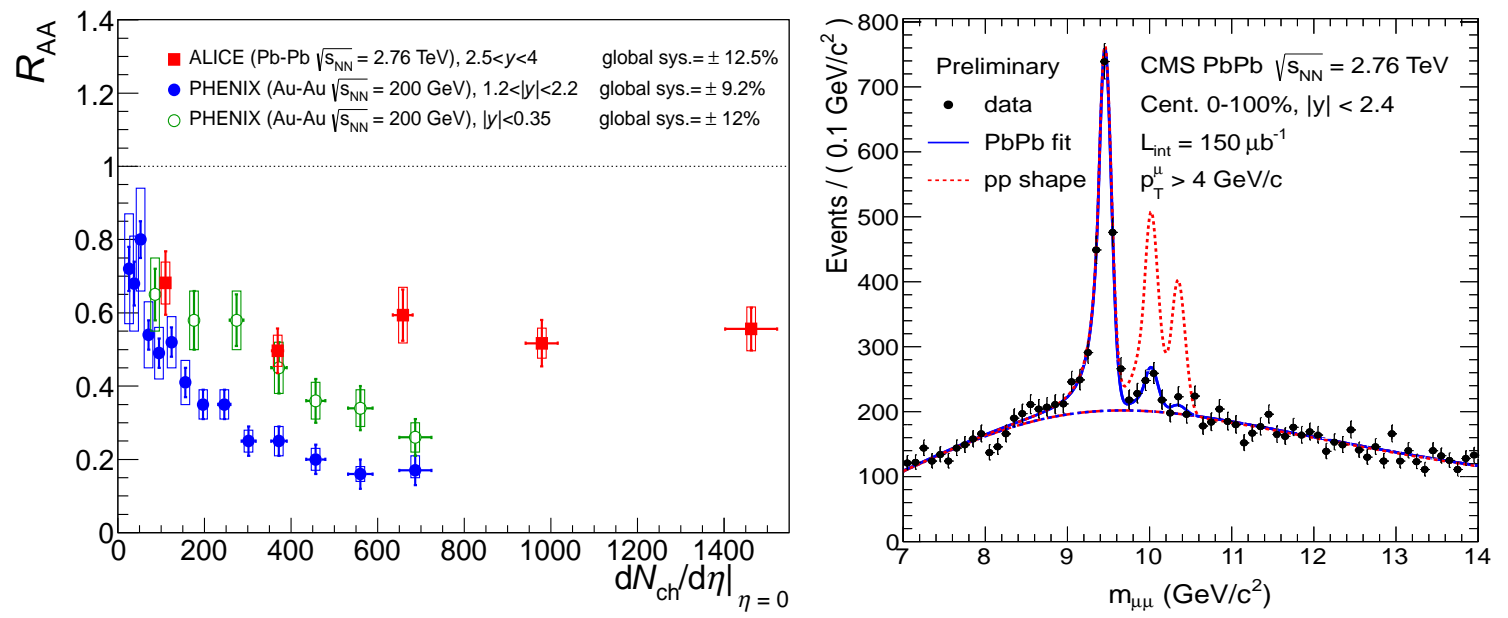

Figure 5: Left: Inclusive $J / \psi R_{A A}$ as a function of the central charged-particle density in $\mathrm{Pb}-\mathrm{Pb}$ at $2.76 \mathrm{TeV}$ compared to PHENIX results in $\mathrm{Au}-\mathrm{Au}$ at $200 \mathrm{GeV}$ at central and forward rapidities [41]. Right: $\Upsilon(\mathrm{nS})$ states measured in $\mathrm{Pb}-\mathrm{Pb}$ (data points) compared to the fitted p-p distribution (dashed red line) at 2.76 $\mathrm{TeV}$ [42].

unique opportunity to study also the dissociation of the $b$-quark bound states. The $\Upsilon$ is expected to survive up to $4 T_{\text {crit }}$ whereas the less tightly-bound $\Upsilon^{\prime}$ and $\Upsilon^{\prime \prime}$ resonances should "melt" at lower temperatures. The data confirms that the $\Upsilon$ states are suppressed in central $\mathrm{Pb}-\mathrm{Pb}$ relative to $\mathrm{p}-\mathrm{p}$ collisions: $R_{A A}(\Upsilon) \approx 0.56, R_{A A}\left(\Upsilon^{\prime}\right) \approx 0.12$ and $R_{A A}\left(\Upsilon^{\prime \prime}\right)<0.1$ (Fig. 5, right) [43, 42]. The deficit in the $\Upsilon$ production yields coincides with the expected fraction from the feed-down contributions of the heavily-suppressed $\Upsilon(2 S, 3 S)$ excited states. The overall scenario is consistent with sequential suppression of the bottomonium family.

\section{Summary}

The main findings in the perturbative sector of $\mathrm{Pb}-\mathrm{Pb}$ collisions at $\sqrt{s_{N N}}=2.76 \mathrm{TeV}$ after 2 years of operation at the LHC can be summarised as follows: 
- Electroweak probes $(\gamma, \mathrm{W}, \mathrm{Z})$ unaffected by final-state interactions can provide, with larger datasets and reduced uncertainties, valuable constraints of the nuclear PDFs $[8,9,10,11,12]$.

- High- $p_{T}$ (leading) hadron production is suppressed compared to p-p collisions by factors ranging from 6 (at $7 \mathrm{GeV} / \mathrm{c}$ ) to 2 (above $40 \mathrm{GeV} / \mathrm{c}$ ), indicating the formation of a dense opaque medium that suppresses the energy of the parent fragmenting partons $[16,17,18]$.

- Fully reconstructed jets are quenched in dijet and $\gamma$-jet events and show (i) a large $p_{T}$ imbalance, (ii) enhanced soft off-cone radiation, (iii) preserved back-to-back azimuthal correlations, and (iv) vacuum-like fragmentation functions (reconstructed using the quenched jet energy) [5, 6, 21, 25, 26, 27], which suggest that parton energy loss occurs via soft multigluon emission followed by vacuum fragmentation.

- $\mathrm{D}$ and $\mathrm{B}$ mesons are suppressed by factors of $\sim 4$ and $\sim 3$ respectively relative to p-p collisions $[19,20]$, in agreement with the expected mass and colour-charge dependencies of radiative energy loss.

- The deficit of $J / \psi$ yields at the LHC is smaller than at lower energies. The suppression is weaker (stronger) at low (high) $p_{T}[9,20,41]$ and is suggestive of model predictions based on colour screening followed by $c \bar{c}$ recombination.

- The $\Upsilon^{\prime}$ and $\Upsilon^{\prime \prime}$ states are strongly suppressed, whereas the $\Upsilon$ ground-state yield is depleted by about $40 \%$ (the same fraction expected from feed-down contribution of the two excited states) relative to p-p collisions $[43,42]$, in agreement with sequential dissociation scenarios.

Figure 3 (left) compiles the $R_{A A}\left(p_{T}\right)$ factors for most of the high- $p_{T}$ and large-mass particles measured in central $\mathrm{Pb}-\mathrm{Pb}$ collisions at the LHC. The mechanisms of initial production of all particles are well understood within $\mathrm{pQCD}$. The final reduced yields observed for all particles containing colour degrees of freedom clearly point to strong final-state effects (radiative energy loss, colour screening, ...). Final extraction of the thermodynamical and transport properties of the hot and dense matter produced at the LHC will require detailed calculations including mechanisms of particle production+"destruction" coupled with a (3D viscous) hydrodynamics description of the expansion of the system.

Acknowledgments: I am grateful to Bernard Pire and Michel Garçon for their invitation to this well-organised and interesting multidisciplinary conference.

\section{References}

[1] S. Chatrchyan et al. [CMS Collaboration], arXiv:1205.2488 [nucl-ex]

[2] F. Karsch, Lect. Notes Phys. 583 (2002) 209

[3] E. V. Shuryak, Sov. Phys. JETP 47 (1978) 212 [Zh. Eksp. Teor. Fiz. 74 (1978) 408]

[4] D. d'Enterria, J. Phys. G 34 (2007) S53

[5] G. Aad et al. [Atlas Collaboration], Phys. Rev. Lett. 105 (2010) 252303

[6] S. Chatrchyan et al. [CMS Collaboration], arXiv:1205.0206 [nucl-ex] 
[7] K. J. Eskola, H. Paukkunen and C. A. Salgado, JHEP 0904 (2009) 065

[8] S. Chatrchyan et al. [CMS Collaboration], Phys. Lett. B 710 (2012) 256

[9] G. Aad et al. [Atlas Collaboration], Phys. Lett. B 697 (2011) 294

[10] S. Chatrchyan et al. [CMS Collaboration], Phys. Rev. Lett. 106 (2011) 212301

[11] Atlas Collaboration, ATLAS-CONF-2011-052.

[12] S. Chatrchyan et al. [CMS Collaboration], arXiv:1205.6334 [nucl-ex]

[13] J. D. Bjorken, FERMILAB-PUB-82-059-THY (1982)

[14] K. Adcox et al. [PHENIX Collaboration], Nucl. Phys. A 757 (2005) 184

[15] J. Adams et al. [STAR Collaboration], Nucl. Phys. A 757 (2005) 102

[16] K. Aamodt et al. [ALICE Collaboration], Phys. Lett. B 696 (2011) 30

[17] H. Appelshauser [ALICE Collaboration], J. Phys. G 38 (2011) 124014

[18] S. Chatrchyan et al. [CMS Collaboration], Eur. Phys. J. C 72 (2012) 1945

[19] B. Abelev [ALICE Collaboration], arXiv:1203.2160 [nucl-ex]

[20] S. Chatrchyan et al. [CMS Collaboration], JHEP 1205 (2012) 063

[21] Atlas Collaboration, ATLAS-CONF-2011-075

[22] J. G. Milhano, EPJ Web Conf. 28 (2012) 03001

[23] N. Armesto, M. Cacciari, T. Hirano, J. L. Nagle and C. A. Salgado, J. Phys. G 37 (2010) 025104

[24] M. Cacciari, J. Rojo, G. P. Salam and G. Soyez, Eur. Phys. J. C 71 (2011) 1539

[25] S. Chatrchyan et al. [CMS Collaboration], Phys. Rev. C 84 (2011) 024906

[26] S. Chatrchyan et al. [CMS Collaboration], Phys. Lett. B 712 (2012) 176

[27] S. Chatrchyan et al. [CMS Collaboration], arXiv:1205.5872 [nucl-ex]

[28] T. Sjostrand, S. Mrenna and P. Z. Skands, JHEP 0605 (2006) 026

[29] Y. L. Dokshitzer and D. E. Kharzeev, Phys. Lett. B 519 (2001) 199

[30] A. Adare et al. [PHENIX Collaboration], Phys. Rev. C 84 (2011) 044905

[31] B. I. Abelev et al. [STAR Collaboration], Phys. Rev. Lett. 106 (2011) 159902

[32] P. Gossiaux, J. Aichelin and T. Gousset, Prog. Theor. Phys. Suppl. 193 (2012) 110

[33] T. Matsui and H. Satz, Phys. Lett. B 178 (1986) 416

[34] S. Datta, F. Karsch, P. Petreczky and I. Wetzorke, Phys. Rev. D 69 (2004) 094507

[35] B. Alessandro et al. [NA50 Collaboration], Eur. Phys. J. C 39 (2005) 335

[36] A. Adare et al. [PHENIX Collaboration], Phys. Rev. Lett. 98 (2007) 232301

[37] A. Adare et al. [PHENIX Collaboration], Phys. Rev. C 84 (2011) 054912

[38] P. Braun-Munzinger and J. Stachel, Phys. Lett. B 490 (2000) 196

[39] R. L. Thews, M. Schroedter and J. Rafelski, Phys. Rev. C 63 (2001) 054905

[40] A. Andronic, P. Braun-Munzinger, K. Redlich and J. Stachel, Phys. Lett. B 652 (2007) 259

[41] B. Abelev et al. [ALICE Collaboration], arXiv:1202.1383 [hep-ex]

[42] T. Dahms [CMS Collaboration], Proceeds. Hard Probes'12, CMS-PAS-HIN-11-011

[43] S. Chatrchyan et al. [CMS Collaboration], Phys. Rev. Lett. 107 (2011) 052302 